\title{
A espiritualidade nos currículos das escolas médicas da região norte e a visão do interno de medicina sobre sua importância na formação
}

\author{
Spirituality in the curriculum of medical schools of \\ northern region and medicine interns vision on its \\ importance in graduation
}

\author{
Simone Regina Souza da Silva Conde', Luis Eduardo de Carvalho Barros', \\ José Heitor Borburema de Oliveira', Ulisses Tavares de Arruda', \\ Sylvia Helena Souza da Silva Batista², Nildo Alves Batista ${ }^{2}$ \\ 'Faculdade de Medicina, Instituto de Ciências da Saúde, Universidade Federal do Pará, Belém (PA), Brasil. \\ ${ }^{2}$ Pós-graduação em Ensino de Ciências da Saúde, Universidade Federal de São Paulo, São Paulo (SP), Brasil.
}

Recebido: Fev. 28, 2018 Aceito: Maio 3, 2018

\section{COMO CITAR ESTE ARTIGO} Conde SRSSC, Barros LEC, Oliveira JHB, Arruda UT, Batista SHSS, Batista NA A espiritualidade nos currículos das escolas médicas da região norte e a visão do interno de medicina so visaso do in Health Education. 2019 Jan-Dez;4(1-2):9-18 https://doi.org/10.4322/ijhe.2018.013

\section{CORRESPONDÊNCIA}

Simone Regina Souza da Silva Conde Faculdade de Medicina, Instituto de Ciências da Saúde, Universidade Federal do Pará

Avenida Generalissimo Deodora, 1 , Bairro Umarizal, CEP 66050-380, Belém (PA), Brasil

sconde@ufpabr

FONTE DE FINANCIAMENTO Edital 03/2015 PIBIC PROPESP UFPA

CONFLITO DE INTERESSE

Os autores declararam não

haver conflitos de interesse.

O estudo foi realizado no Centro de Desenvolvimento do Ensino Superior em Saúde (CEDESS), Universidade Federal de São Paulo (SP), Brasil.

Todos os autores leram e aprovam versão final submetida ao Interdisciplinary Journal of Health Education (IJHE).

\section{RESUMO}

Introdução: O cuidado ampliado à saúde deve incluir o bem-estar espiritual. No Brasil, estudos prévios demonstram pouca inserção da espiritualidade nos currículos das áreas de saúde. Objetivo: Este estudo investigou a presença desta temática nos currículos das escolas médicas da região Norte do país e analisou a visão do interno de medicina sobre a importância do tema e seu próprio bem-estar espiritual. Método: Desenvolvida pesquisa do tipo transversal e descritivo, explorando projetos pedagógicos de dezessete escolas médicas; acrescido da aplicação de duas escalas tipo likert a 92 graduandos do último semestre de medicina, oriundos duas escolas médicas da região. A primeira escala versou sobre três dimensões (Espiritualidade e Saúde, Espiritualidade e Doença, Espiritualidade e Formação Médica), com pontuações de 1 a 4 . As médias das assertivas foram divididas em três intervalos de pontuação: de 1 a 1,99 - zona de perigo; de 2 a 2,99 - zona de alerta; e de 3 a 4 - zona de conforto, conforme o comportamento atitudinal do grupo. Após a aplicação do questionário houve o processo de validação e de confiabilidade da mesma. A segunda escala se referiu ao bem-estar espiritual com o uso do escore FACIT-Sp-Non-IIInes - Version4, entre 0 a 48 pontos. Resultados: Dezesseis instituições responderam ao questionário e em $12,5 \%$ se identificou a presença da temática espiritualidade, em seus currículos. As médias dos escores das dimensões espiritualidade e saúde, espiritualidade e doença e espiritualidade e formação foram 3,29; 3,47 e 2,61, respectivamente, revelando fragilidade no aspecto de formação. O escore final do bem-estar espiritual dos graduandos dos cursos foi de 37 pontos. Conclusão: Concluiu-se que há baixa inserção da espiritualidade nos currículos, e, apesar dos graduandos estarem em bem-estar espiritual e reconhecerem sua importância, há grandes lacunas em sua formação.

PALAVRAS-Chave: Currículo. Espiritualidade. Educação de graduação em medicina.

\section{ABSTRACT}

Introduction: Extended health care should include spiritual well-being. In Brazil, previous studies show little insertion of spirituality in the curricula of health areas. Objective: this study investigated the presence of this theme in the curricula of the medical schools of the North region of the country and analyzed the medical intern's view on the importance of the theme and his own spiritual well-being. Methods: developed transversal and descriptive research, exploring pedagogical projects of seventeen medical schools; plus the application of two likert-type scales to 92 graduates of the last semester of medicine, from two medical schools in the region. The first scale was based on three dimensions (Spirituality and Health, Spirituality and Disease, Spirituality and Medical Formation), with scores of 1 to 4 . The averages of the assertions were divided into three scoring intervals: from 1 to 1.99 - zone of Danger; from 2 to 2,99 - alert zone; and 3 to 4 - comfort zone, according to the attitudinal behavior of the group. After the application of the questionnaire, there was the process of validation and reliability of the same. The second scale referred to spiritual well-being using the FACIT-Sp-Non-IIInes - Version4 score, from 0 to 48 points. Results: sixteen institutions answered the questionnaire and in $12.5 \%$ the presence of the spirituality theme was identified in their curricula. The average scores of the dimension's spirituality and health, spirituality 
and disease and spirituality and training were 3.29; 3.47 and 2.61, respectively, revealing fragility in the forming aspect. The final spiritual well-being score of the graduates of the courses was 37 points. Conclusion: It was concluded that there is a low insertion of spirituality in the curricula, and although the students are in spiritual well-being and recognize its importance, there are great gaps in their formation.

KEYWORDS: Curriculum. Spirituality. Education, Medical, Undergraduate.

\section{Introdução}

A mais recente definição de saúde proposta pela Organização Mundial de Saúde (OMS) propõe que se trata de um dinâmico estado de bem-estar físico, mental, social, ambiental e espiritual e não meramente a ausência da doença, incluindo a espiritualidade, a religiosidade e as crenças pessoais nos instrumentos de avaliação de qualidade de vida ${ }^{1,2}$.

Nota-se um crescente interesse pelas pesquisas que demostrem qual a relação entre o bem estar espiritual e ou religioso e todo o processo de promoção da saúde ${ }^{3}$, do seu impacto sobre o aparecimento de enfermidades ${ }^{4}$, sobre sua participação no resultado de diversos tratamentos ${ }^{5}$, e em especial, sobre a qualidade de vida do ser humano enfermo ou não ${ }^{6}$. Na maioria destes estudos, conseguiu-se demonstrar por meio de diversas escalas, uma relação positiva entre o melhor grau de bem-estar espiritual e ou religioso com uma melhor qualidade de vida, mesmo diante de doenças crônico-degenerativas e oncológicas ${ }^{7,8}$. Em outros, com múltiplos desenhos metodológicos, observou-se uma associação, um pouco mais fraca, entre a espiritualidade e religiosidade e alterações bioquímicas, endocrinológicas e imunológicas9.

Em que pese, a espiritualidade e a religiosidade serem questões relevantes na vida do ser humano e ser uma dimensão importante a ser considerada na busca da saúde, pouca importância se dá, de forma intencional, nos currículos das escolas médicas do Brasil. Estudo nesta linha ${ }^{10}$ revelou que 10,4\% de 86 escolas brasileiras pesquisadas possuíam cursos de Saúde e Espiritualidade, todas de carácter eletivo. Este panorama difere do encontrado nas escolas americanas e do Reino Unido, onde $90 \%$ e $59 \%$, respectivamente, possuem momentos específicos sobre esta temática em seus currículos ${ }^{11,12}$.

Estes últimos dados respondem às orientações da Association of American Medical Colleges (AAMC), da Joint Commission on Accreditation of Healthcare Organizations (JCAHO) e da OMS que recomendam que a espiritualidade deva fazer parte da formação médica, em publicação de quase duas décadas atrás ${ }^{13}$.

Há poucos dados sobre este assunto nos cursos médicos da região Norte do país. Ressaltam-se as características peculiares marcantes quanto aos aspectos étnicos, culturais e antropológicos, destacando-se, neste contexto, a forte influência da medicina popular e da pajelança em várias comunidades da região.

Este trabalho pretendeu identificar a presença formal da espiritualidade nos currículos das escolas médicas da região Norte, assim como analisar, sob a ótica dos internos de medicina, a importância da espiritualidade como parte do cuidado integral do ser humano, no contexto de sua formação médica, além de seu próprio bem-estar espiritual.

\section{Método}

\section{Delineamento e população de estudo}

Tratou-se de um estudo transversal, descritivo e analítico, com abordagens quantitativas, desenvolvida com dezessete projetos pedagógicos dos cursos de medicina da região Norte, que no mínimo formaram uma turma até o final de 2014, credenciadas pelo Ministério da Saúde do Brasil. A segunda população de pesquisa 
se constituiu do universo dos alunos do último semestre do curso de medicina, pertencentes a duas escolas médicas, localizadas na cidade de Belém, capital do estado do Pará.

Esta pesquisa foi realizada segundo os preceitos éticos vigentes no país (Resolução 466/2012), recebendo a aprovação do Comitê de Ética em Pesquisa de Seres Humanos da Universidade Federal de São Paulo (CEP - UNIFESP), sob o registro do CAAE 44073115.8.0000.5505. Todos os sujeitos da pesquisa assinaram o Termo de Consentimento Livre e Esclarecido.

Procedimentos de produção de dados

Pesquisa documental

A pesquisa documental foi feita nos projetos pedagógicos publicados nos sites das instituições selecionadas, envio de questionários eletrônicos e posterior contato telefônico com os coordenadores de cada curso para confirmar as informações colhidas e obter maior detalhamento das mesmas.

A escala de reconhecimento da importância dada pelos internos de medicina sobre a abordagem da espiritualidade como parte do cuidado integral do ser humano foi do tipo likert composta de três dimensões (Espiritualidade e Saúde, Espiritualidade e Doença e Espiritualidade e Formação Acadêmica) com 25 assertivas ao total. Para cada assertiva, as opções de resposta foram concordo totalmente, inclinado a concordar, inclinado a discordar e discordo totalmente, pontuando de 1 a 4 .

A escala de bem-estar espiritual dos internos de Medicina se constituiu da versão para o português do escore Functional Assessment of Chronic Illness Therapy - Spiritual Well-Being, a modified version for non-illness - FACIT-Sp-Non-Illness - Version 4. Esta escala tipo likert, contém duas dimensões, sentimento/paz e fé, com doze assertivas no total e escores variando de 0 a 48 pontos.

Análise dos dados

Análise dos dados da pesquisa documental

Para a análise documental, foram utilizados os bancos de dados dos programas Epi Info 7.1.4 e Excel do Windows 2013, empregando-se a estatística descritiva.

Análise da escala de reconhecimento da importância dado pelos internos de medicina sobre a abordagem da espiritualidade como parte do cuidado integral do ser humano.

Em um primeiro momento, houve um pré-teste com 15 graduandos de medicina do $10^{\circ}$ período, para avaliar a fraseologia e o tempo de preenchimento da escala. Posteriormente, foi realizada a análise de validação e de confiabilidade das assertivas ${ }^{14}$. A confiabilidade do instrumento foi calculada a partir do coeficiente de correlação, envolvendo o total de pontos por respondente entre a primeira e a segunda aplicação, conhecida como coeficiente de confiabilidade e o método dito teste-reteste ${ }^{15}$, sendo o critério de aceitação um mínimo de $80 \%$, equivalendo a $\mathrm{R}$ igual ou maior que 0,80 .

$\mathrm{Na}$ análise final, as médias das assertivas foram divididas em três intervalos de pontuação: de 1 a 1,99 - zona de perigo; de 2 a 2,99 - zona de alerta; e de 3 a 4 - zona de conforto, conforme o comportamento atitudinal do grupo.

Utilizou-se a planilha eletrônica MS-Excel, em sua versão do MS-Office 2010, para a organização dos dados, e o pacote estatístico IBM SPSS (Statistical Package for Social Sciences), em sua versão 23.0, para a obtenção dos resultados.

Análise da escala FACITsp-12 versão 4.

A análise do questionário FACITsp-12 for Non-Illness obedeceu às orientações do FACITsp Scoring Guidlines (version 4), baseada no peso de cada assertiva, retirando uma média da dimensão de sentimento/paz (escores de 0 a 32) e de fé (escores de 0 a 16), 
com escore total variando de 0 a 48 pontos. O processo de validação e interpretação da escala FACITsp-12 versão 4 foi realizada previamente para a língua inglesa e disponibilizado no site www.facit.org, nos programas SPSS e SAS.

Para correlacionar a média dos escores das duas escalas utilizadas, foi utilizado o método de correlação de Spearman bivariada ${ }^{8}$.

\section{Resultados}

Das dezessete escolas médicas selecionadas, dezesseis (94\%) responderam ao questionário proposto, cujos principais resultados constam na tabela 1.

Observou-se um total de 1.442 vagas de ingressantes anuais, maioria composta de escolas públicas (62,5\%); oito (50\%) declararam utilizar metodologias ativas de ensino e aprendizagem como a metodologia predominante; e duas estarem em processo de transição do modelo tradicional para o ativo.

Tabela 1. Características das escolas médicas da região norte pesquisadas e a presença da temática saúde e espiritualidade no currículo formal.

\begin{tabular}{|c|c|c|c|c|}
\hline Estado & $\begin{array}{l}\text { Cidade } \\
\text { Natureza }\end{array}$ & Instituição & $\begin{array}{l}\text { Ano de Início } \\
\text { Alunos } \\
\text { Ingressantes } \\
\text { Anual }\end{array}$ & $\begin{array}{c}\text { Presença da } \\
\text { temática Saúde e } \\
\text { Espiritualidade no } \\
\text { Projeto Pedagógico }\end{array}$ \\
\hline Acre & $\begin{array}{l}\text { Rio Branco } \\
\text { Pública }\end{array}$ & Universidade Federal do Acre & $\begin{array}{c}2002 \\
80\end{array}$ & Não \\
\hline \multirow{3}{*}{ Amazonas } & $\begin{array}{l}\text { Manaus } \\
\text { Privada }\end{array}$ & Centro Universitário Nilton Lins & $\begin{array}{c}2006 \\
100\end{array}$ & Não \\
\hline & $\begin{array}{l}\text { Manaus } \\
\text { Pública }\end{array}$ & $\begin{array}{l}\text { Universidade do Estado do } \\
\text { Amazonas }\end{array}$ & $\begin{array}{c}2001 \\
120\end{array}$ & Não \\
\hline & $\begin{array}{l}\text { Manaus } \\
\text { Pública }\end{array}$ & $\begin{array}{l}\text { Universidade Federal do } \\
\text { Amazonas }\end{array}$ & $\begin{array}{c}1965 \\
112\end{array}$ & Não \\
\hline \multirow{4}{*}{ Pará } & $\begin{array}{l}\text { Belém } \\
\text { Privada }\end{array}$ & $\begin{array}{l}\text { Centro Universitário do estado do } \\
\text { Pará }\end{array}$ & $\begin{array}{c}2007 \\
100\end{array}$ & Sim \\
\hline & $\begin{array}{l}\text { Belém } \\
\text { Pública }\end{array}$ & Universidade Estadual do Pará & $\begin{array}{c}1971 \\
100\end{array}$ & Não \\
\hline & $\begin{array}{l}\text { Santarém } \\
\text { Pública }\end{array}$ & Universidade Estadual do Pará & $\begin{array}{c}2006 \\
40\end{array}$ & Não \\
\hline & $\begin{array}{l}\text { Belém } \\
\text { Pública }\end{array}$ & Universidade Federal do Pará & $\begin{array}{c}1919 \\
150\end{array}$ & Não \\
\hline \multirow{3}{*}{ Rondônia } & $\begin{array}{l}\text { Cacoal } \\
\text { Privada }\end{array}$ & $\begin{array}{l}\text { Faculdade de Ciências Biomédicas } \\
\text { de Cacoal }\end{array}$ & $\begin{array}{c}2007 \\
50\end{array}$ & Não \\
\hline & $\begin{array}{l}\text { Porto Velho } \\
\text { Privada }\end{array}$ & Faculdade de São Lucas & $\begin{array}{c}2004 \\
90\end{array}$ & Não \\
\hline & $\begin{array}{l}\text { Porto Velho } \\
\text { Pública }\end{array}$ & $\begin{array}{l}\text { Fundação Universidade Federal } \\
\text { de Rondônia }\end{array}$ & $\begin{array}{c}2002 \\
40\end{array}$ & Não \\
\hline Roraima & $\begin{array}{l}\text { Boa Vista } \\
\text { Pública }\end{array}$ & Universidade Federal de Roraima & $\begin{array}{c}1993 \\
80\end{array}$ & Não \\
\hline \multirow{4}{*}{ Tocantins } & $\begin{array}{l}\text { Gurupi } \\
\text { Pública }\end{array}$ & Centro Universitário de Gurupi & $\begin{array}{c}2002 \\
120\end{array}$ & Não \\
\hline & $\begin{array}{l}\text { Araguaia } \\
\text { Privada }\end{array}$ & $\begin{array}{l}\text { Faculdade de Ciências Humanas, } \\
\text { Econômicas e da Saúde de } \\
\text { Araguaia }\end{array}$ & $\begin{array}{c}1999 \\
60\end{array}$ & Não \\
\hline & $\begin{array}{l}\text { Porto } \\
\text { Nacional } \\
\text { Privada }\end{array}$ & $\begin{array}{l}\text { Fundação Presidente Antonio } \\
\text { Carlos }\end{array}$ & $\begin{array}{c}2004 \\
120\end{array}$ & $\operatorname{Sim}$ \\
\hline & $\begin{array}{l}\text { Palmas } \\
\text { Pública }\end{array}$ & Universidade Federal do Tocantins & $\begin{array}{c}2008 \\
80\end{array}$ & Não \\
\hline
\end{tabular}


A presença da temática espiritualidade foi declarada no currículo de duas instituições $(12,5 \%)$, sendo ambas de natureza privada. Em uma das escolas, esta abordagem se faz de modo transversal, com atividades predominantemente teóricas. Na segunda escola, o coordenador não informou como se dá o desenvolvimento metodológico desta unidade. Em uma terceira instituição, há uma proposta de introdução desta temática como disciplina eletiva e sendo uma das linhas de pesquisa no novo projeto pedagógico em construção e com previsão de implantação para breve.

Em relação aos estudantes de medicina, foram aplicados 92 instrumentos de pesquisa com escalas tipo likert aos graduandos do último período, de um universo populacional de 96 alunos (95,8\%).

A tabela 2 demonstra algumas características demográficas desta população, observando-se um grupo de predomínio de homens $(54,3 \%)$, com faixa etária entre os 20-25 anos (53,2\%), morando com os familiares $(68,5 \%)$ e maioria declarou praticar religião $(87 \%)$. Entre os que possuem religião, a católica foi a predominante $(72,5 \%)$, seguida evangélica $(17,5 \%)$ e da espírita (5\%). No grupo sem religião, total de doze alunos, seis se declararam agnósticos e dois ateus.

Tabela 2. Características demográficas dos graduandos de medicina de duas escolas da região Norte do país.

\begin{tabular}{lc}
\multicolumn{1}{c}{ Variáveis } & $\mathbf{N}(\%)$ \\
Gênero & $50(54,3)$ \\
Masculino & $42(45,7)$ \\
$\quad$ Feminino & \\
\hline Faixa Etária (anos) & $49(53,2)$ \\
$20-25$ & $33(35,9)$ \\
$26-31$ & $10(10,9)$ \\
Acima de 31 & \\
\hline Procedência & $80(86,9)$ \\
Norte & $10(10,9)$ \\
Nordeste & $2(2,2)$ \\
Sudeste & \\
\hline Com quem mora & $63(68,5)$ \\
Família & $17(18,5)$ \\
Sozinho & $8(8,7)$ \\
Cônjuge & $4(4,3)$ \\
Amigo & \\
\hline Possui filhos & $79(87,3)$ \\
Não & $13(12,7)$ \\
Sim & \\
\hline Possui religião & $80(87 \%)$ \\
Sim & $12(13 \%)$ \\
Não &
\end{tabular}

A análise dos resultados da escala de reconhecimento da importância dado pelos internos de medicina sobre a abordagem da espiritualidade como parte do cuidado integral do ser humano resultou na validação de 20 das 25 assertivas com confiabilidade de 0,82 . A partir das assertivas validadas, as médias gerais das dimensões pesquisadas estão dispostas na figura 1 . Nesta, observou-se que há uma clara percepção dos alunos sobre a influência da espiritualidade sobre o estado de saúde e de doença do indivíduo, por suas médias se encontrarem em zona de conforto, mas quanto ao aspecto de formação, a média se encontra em zona de alerta.

As tabelas 3, 4 e 5 apresentam os resultados encontrados nas dimensões Espiritualidade e Saúde, Espiritualidade e Doença e Espiritualidade e Formação Acadêmica, respectivamente, com suas assertivas validadas. 


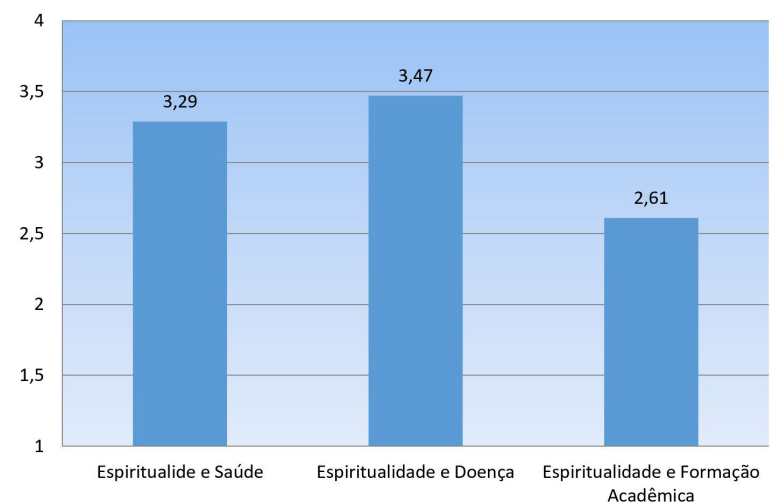

Figura 1. Médias gerais das dimensões Espiritualidade e Saúde, Espiritualidade e Doença e Espiritualidade e Formação Acadêmica, de acordo com assertivas validadas no instrumento de escala atitudinal aplicado a graduandos de medicina da região Norte do país.

Tabela 3. Resultados da escala atitudinal na Dimensão Espiritualidade e Saúde com suas respectivas assertivas e médias.

\begin{tabular}{|c|c|c|c|c|c|}
\hline \multirow{2}{*}{ Assertivas } & \multicolumn{4}{|c|}{ Escala Atitudinal } & \multirow{2}{*}{$\begin{array}{l}\text { Média } \\
+ \text { DP }\end{array}$} \\
\hline & СТ $\%$ & IC $\%$ & ID $\%$ & DT $\%$ & \\
\hline $\begin{array}{l}\text { A saúde é um estado de bem-estar físico, mental, social } \\
\text { e espiritual. }\end{array}$ & 78,3 & 19,6 & 1,1 & 1,1 & $3,75+0,53$ \\
\hline $\begin{array}{l}\text { O bem - estar espiritual influencia positivamente na } \\
\text { saúde do indivíduo. }\end{array}$ & 71,7 & 26,1 & 2,2 & 0 & $3,70+0,51$ \\
\hline $\begin{array}{l}\text { Acredito ser importante conhecer se meu paciente está } \\
\text { em bem-estar espiritual. }\end{array}$ & 48,9 & 43,5 & 7,6 & 0 & $3,41+0,63$ \\
\hline $\begin{array}{l}\text { Estou preparado para abordar questões de } \\
\text { espiritualidade e crenças pessoais com meu paciente. }\end{array}$ & 14,1 & 41,3 & 37 & 7,6 & $2,62+0,82$ \\
\hline $\begin{array}{l}\text { A pesquisa da dimensão da espiritualidade, da } \\
\text { religiosidade e das crenças espirituais deve fazer parte } \\
\text { dos questionários de qualidade de vida. }\end{array}$ & 32,6 & 62 & 3,3 & 2,2 & $3,25+0,62$ \\
\hline $\begin{array}{l}\text { A crença de que a espiritualidade influencia no estado } \\
\text { de saúde de seu paciente tem relação direta com o } \\
\text { bem-estar espiritual do médico. }\end{array}$ & 28,3 & 51,1 & 15,2 & 5,4 & $3,02+0,81$ \\
\hline
\end{tabular}

CT: Concordo totalmente; IC: Inclinado a concordar; ID: Discordo totalmente; DT: Discordo totalmente; DP: Desvio padrão.

Tabela 4. Resultados da escala atitudinal na Dimensão Espiritualidade e Doença com suas respectivas assertivas e médias.

\begin{tabular}{|c|c|c|c|c|c|}
\hline \multirow{2}{*}{ Assertivas } & \multicolumn{4}{|c|}{ Escala Atitudinal } & \multirow{2}{*}{$\begin{array}{l}\text { Média } \\
+ \text { DP }\end{array}$} \\
\hline & $\mathrm{CT} \%$ & $\mathrm{IC} \%$ & ID $\%$ & DT $\%$ & \\
\hline $\begin{array}{l}\text { O grau de bem-estar espiritual influencia no } \\
\text { desenvolvimento de doença. }\end{array}$ & 62 & 37 & 1,1 & 0 & $3,61+0,51$ \\
\hline $\begin{array}{l}\text { Os aspectos acerca da espiritualidade e das crenças } \\
\text { pessoais devem ser levadas em conta no momento da } \\
\text { escolha do tratamento proposto. }\end{array}$ & 33,7 & 45,7 & 12 & 8,7 & $3,04+0,9$ \\
\hline $\begin{array}{l}\text { Os aspectos da espiritualidade, da religiosidade e das } \\
\text { crenças pessoais influenciam na atitude do paciente em } \\
\text { lidar com sua doença. }\end{array}$ & 79,3 & 18,5 & 2,2 & 0 & $3,77+0,47$ \\
\hline $\begin{array}{l}\text { A fé e a paz interior se associam ao tempo de } \\
\text { recuperação das doenças. }\end{array}$ & 55,4 & 38 & 6,5 & 0 & $3,49+0,62$ \\
\hline $\begin{array}{l}\text { A dimensão espiritual e de crenças pessoais influencia } \\
\text { na qualidade de vida nos portadores de doenças } \\
\text { oncológicas e crônico-degenerativas. }\end{array}$ & 59,8 & 33,7 & 5,4 & 1,1 & $3,52+0,65$ \\
\hline $\begin{array}{l}\text { Os pacientes em cuidados paliativos devem ter suporte } \\
\text { acerca de sua espiritualidade. }\end{array}$ & 62 & 34,8 & 3,3 & 0 & $3,59+0,56$ \\
\hline $\begin{array}{l}\text { Técnicas de suporte espiritual auxiliam para a melhora do } \\
\text { paciente quando aliadas aos tratamentos convencionais. }\end{array}$ & 42,4 & 47,8 & 7,6 & 2,2 & $3,30+0,71$ \\
\hline
\end{tabular}

CT: concordo totalmente; IC: inclinado a concordar; ID: discordo totalmente; DT: discordo totalmente; DP: desvio padrão. 
Tabela 5. Resultados da escala atitudinal na Dimensão Espiritualidade e Formação Acadêmica com suas respectivas assertivas e médias.

\begin{tabular}{|c|c|c|c|c|c|}
\hline \multirow{2}{*}{ Assertivas } & \multicolumn{4}{|c|}{ Escala Atitudinal } & \multirow{2}{*}{$\begin{array}{l}\text { Média } \\
+ \text { DP }\end{array}$} \\
\hline & $\mathrm{CT} \%$ & IC $\%$ & ID $\%$ & DT\% & \\
\hline $\begin{array}{l}\text { O conteúdo espiritualidade e saúde deveria fazer parte } \\
\text { dos currículos dos cursos de medicina. }\end{array}$ & 22,8 & 47,8 & 27,2 & 2,2 & $2,91+0,77$ \\
\hline $\begin{array}{l}\text { Durante o meu curso, houve momento específico para a } \\
\text { abordagem do tema Saúde e Espiritualidade. }\end{array}$ & 6,5 & 18,5 & 22,8 & 52,2 & $1,79+0,97$ \\
\hline $\begin{array}{l}\text { A temática Saúde e Espiritualidade deve ser uma } \\
\text { disciplina obrigatória. }\end{array}$ & 10,9 & 34,8 & 37 & 17,4 & $2,39+0,9$ \\
\hline $\begin{array}{l}\text { A temática Saúde e Espiritualidade deve ser uma } \\
\text { disciplina eletiva. }\end{array}$ & 19,6 & 55,4 & 18,5 & 6,5 & $2,88+0,8$ \\
\hline $\begin{array}{l}\text { Possuo um conhecimento suficiente sobre a Saúde e } \\
\text { Espiritualidade dentro da minha formação profissional } \\
\text { para abordá-la com meus pacientes. }\end{array}$ & 7,6 & 26,1 & 44,6 & 21,7 & $2,2+0,87$ \\
\hline $\begin{array}{l}\text { Adquiri informações sobre saúde e espiritualidade fora } \\
\text { do meu curso de medicina. }\end{array}$ & 31,5 & 41,3 & 8,7 & 18,5 & $2,86+1,07$ \\
\hline $\begin{array}{l}\text { Ter o conhecimento sobre os aspectos científicos da } \\
\text { temática Saúde e Espiritualidade melhora a atuação } \\
\text { profissional médica. }\end{array}$ & 37 & 51,1 & 7,6 & 4,3 & $3,21+0,76$ \\
\hline
\end{tabular}

CT: concordo totalmente; IC: inclinado a concordar; ID: discordo totalmente; DT: discordo totalmente; DP: desvio padrão.

A presente pesquisa encontrou, mediante a aplicação da escala de Bem-Estar Espiritual, o escore geral de 37 pontos, sendo que a dimensão significado/paz alcançou 25 pontos e a de fé 12 pontos.

A correlação dos escores das escalas aplicadas revela uma correlação positiva entre a dimensão Espiritualidade e Saúde e a escala de Bem-Estar Espiritual ( $p: 0,012)$ e indiferente com as dimensões Saúde e Doença $(p: 0,203)$ e Saúde e Formação $(p: 0,291)$.

\section{Discussão}

Em estudos de prevalência acerca da presença da temática espiritualidade nos currículos formais, nas escolas médicas dos $\mathrm{EUA}^{11} \mathrm{e}$ do Reino Unido ${ }^{12}$, os percentuais foram bem maiores, $90 \%$ e $59 \%$, respectivamente, do que os $12,4 \%$ encontrados na atual pesquisa, em instituições privadas e de carácter obrigatório. Em um inquérito sobre a presença da espiritualidade nos currículos das escolas médicas do Brasil ${ }^{10}$, encontraram um percentual de 10,4\%. Em quatro escolas, de carácter privado, o conteúdo foi desenvolvido em disciplinas obrigatórias e em cinco outras, todas públicas, em disciplinas eletivas.

Fazendo um comparativo entre estas informações, observa-se que ainda na graduação médica no Brasil não há uma preocupação clara com a dimensão espiritual do homem e como esta abordagem pode auxiliar no processo de promoção da saúde e nos processos terapêuticos e de reabilitação. Este fato pode ser em decorrência da falta de conhecimento e de aprofundamento no assunto, gerando resistências por não achar comprovação científica ou por acreditar que o suporte espiritual deva ser realizado fora dos ambientes acadêmicos.

Para os graduandos pesquisados, cerca de $98 \%$ concordaram com a definição de saúde da OMS e que o bem-estar espiritual influencia positivamente na saúde do indivíduo, assim como $94 \%$ acreditaram que as dimensões de espiritualidade, de religiosidade e de crenças deveriam fazer parte do questionários de qualidade de vida, sendo menor o percentual, em torno de $68 \%$, dos que se acharam preparados para abordar esta temática com os pacientes.

Na dimensão Espiritualidade e Doença, entre 98 e 99\% dos estudantes concordaram que o bem-estar espiritual influência no aparecimento da doença e como estes 
lidam com os agravos. Dentre eles, também, 79,4\% acreditaram que os aspectos da espiritualidade e das crenças deveriam ser levados em conta na escolha do tratamento proposto e acima de $90 \%$ concordaram que a fé e a paz interior se associam com o tempo de recuperação e que os pacientes com doenças crônico-degenerativas e em cuidados paliativos deveriam ter suporte espiritual, pois auxiliaria quando aliados às terapias convencionais.

Em $70,6 \%$ dos graduandos, houve concordância de que a espiritualidade deveria fazer parte dos currículos dos cursos de medicina, de forma mais eletiva $(75 \%)$ do que obrigatória $(45,7 \%)$. Contudo, a maioria não identificou momentos específicos para esta temática ao longo do seu curso (75\%); adquirindo informações fora da escola médica. Em $72,8 \%$ dos casos, os graduandos não acharam possuir conhecimento suficiente sobre a temática para abordarem com seus pacientes, em 64,3\% das respostas.

Dados muito semelhantes foram descritos em uma outra população de estudantes brasileiros de medicina ${ }^{16}$, onde a maioria também acreditava no impacto da espiritualidade na saúde dos pacientes $(71,2 \%)$ e gostariam de abordar a espiritualidade e a religião na prática clínica $(58 \%)$, contudo se sentiam despreparados para fazê-lo.

Pelos resultados expostos está clara a lacuna que há na formação dos graduandos de Medicina pesquisados sobre a dimensão espiritual do ser humano e como se faz esta abordagem no âmbito do cuidado integral. Por ser esta dimensão identificada em conjunto com a física, a mental e a social como essenciais à saúde humana pela $\mathrm{OMS}^{1}$, como estimular seu emprego?

Em um estudo de revisão sobre a temática ${ }^{17}$, identificaram um interesse por parte dos pacientes em que seus médicos lhe perguntassem sobre suas crenças espirituais em torno de $74 \%$. Por outro lado, isto só era realizado em $15,6 \%$ dos atendimentos.

Em consonância com a necessidade do cuidado centrado na pessoa exigir a observância de todas suas dimensões, várias renomadas instituições passaram a recomendar a introdução da temática espiritualidade na formação dos profissionais de saúde, a destacar a OMS, a AAMC, a JCAHO e o Americam College of Physicians ${ }^{18}$.

Investigando como países europeus introduziram a temática espiritualidade na educação ao cuidado ${ }^{19}$, encontrou-se em essa abordagem em várias áreas de saúde, como a medicina, a enfermagem, a psicologia e equipes multidisciplinares. Nestas a temática estava incluída em diversas unidades curriculares, entre as quais na comunicação de más notícias, no estudo de influência de histórias e fatos da vida pessoal no desenvolvimento de doenças, nos cuidados paliativos, no aconselhamento psicológico e social, na abordagem da ética, no desenvolvimento de linhas de pesquisa, entre outros.

Neste sentido, várias publicações surgiram com o intuito orientar a introdução da espiritualidade nos currículos de saúde, como a abordagem da anamnese espiritual ${ }^{20}$, do uso da religião, da espiritualidade e da fé para lidar com o estresse e com as consequências dos problemas da vida, o chamado Coping Espiritual / Religioso ${ }^{21}$, de instrumentos técnicos que orientam acessar a história espiritual do paciente ${ }^{22}$, assim como integrando a espiritualidade de modo sólido no cuidado ao paciente ${ }^{3}$.

No atual estudo, o grupo de graduandos pesquisados possuía um alto nível de bem-estar espiritual e ficou demonstrado, nos resultados anteriores, que é uma população que reconhece a importância da espiritualidade sobre a saúde e a doença, apesar de fraca formação acadêmica na área.

Alguns autores ${ }^{23,24}$ afirmam que somente os profissionais que se preocupam com sua própria espiritualidade são mais propensos a conferir importância desta dimensão em sua prática profissional.

Independente da crença ou não pessoal, não há como deixar de reconhecer que o Brasil é um país com $90 \%$ de população religiosa e este lado precisa ser levado em conta no momento de pensar nas políticas de promoção à saúde, seja individual ou coletiva, de forma científica e cuidadosa. Os diagnósticos situacionais estão postos, 
incluindo o do presente estudo, necessitando avançar no desenvolvimento crescente dos estímulos à sensibilização do corpo docente acerca da necessidade da introdução desta temática na formação dos profissionais de saúde.

\section{Conclusões}

A análise dos resultados demonstrados revelou que entre as escolas médicas da região norte investigadas, uma minoria possui a temática espiritualidade inserida em seus currículos, sendo estas, instituições privadas. Destacando que uma descreveu esta inserção como diluída em vários momentos do eixo transversal.

Do total de graduandos de medicina pesquisados, houve uma boa percepção da importância da relação de espiritualidade e saúde e de espiritualidade e doença, porém não ocorreu a formação acadêmica na área de forma adequada.

Pela metodologia empregada, o grupo de estudantes foi classificado como de alto nível de bem-estar espiritual, com maioria religiosa e houve correlação positiva entre este bem-estar e a dimensão que relaciona espiritualidade e saúde.

\section{Agradecimentos}

Agradecemos aos coordenadores dos cursos de Medicina das instituições pesquisadas e ao programa PIBIC - FAPESPA / UFPA.

\section{Referências}

1. World Health Organization (WHO). WHOQOL and spirituality, religiousness and personal beliefs (SRPB): Report on WHO Consultation. Geneva: WHO; 1998. pp. 2-23.

2. WHOQOL SRPB GROUP. A cross-cultural study of spirituality, religion, and personal beliefs as components of quality of life. Soc Sci Med. 2005;62(6):1486-97. PMid:16168541.

3. Puchalski CM. Integrating spirituality into patient care: An essential element of person-centered care. Pol Arch Med Wewn. 2013;123(9):491-7. http://dx.doi.org/10.20452/pamw.1893. PMid:24084250.

4. Wachholtz A, Rogoff M. The relationship between spirituality and burnout among medical students. Changes. 2012;29(2):997-1003.

5. Evangelista CB, Lopes MEL, Costa SFG, Batista PCS, Batista JBV, Oliveira AMM. Cuidados paliativos e espiritualidade: revisão integrativa da literatura. Rev Bras Enferm. 2016;69(3):554-63. http://dx.doi.org/10.1590/0034-7167.2016690324i. PMid:27355311.

6. Davison SN, Jhangri GS. Existential and religious dimensions of spirituality and their relationship with health-related quality of life in chronic kidney disease. Clin J Am Soc Nephrol. 2010;5(24):1969-76. http://dx.doi.org/10.2215/CJN.01890310. PMid:20651152.

7. Bentley B, O'Connor M, Kane R, Breen LJ. Feasibility, acceptability, and potential effectiveness of dignity therapy for people with motor neurone disease. PLoS One. 2014;9(5):1-7. http://dx.doi.org/10.1371/journal.pone.0096888. PMid:24816742.

8. Lewis S, Salins N, Rao MR, Kadam A. Spiritual well-being and its influence on fatigue in patients undergoing active cancer directed treatment: A correlational study. J Cancer Res Ther. 2014;10(3):676-81. PMid:25313759.

9. Schettino JR, Olmos NT, Myers HF, Joseph NT, Poland RE, Lesser IM. Religiosity and treatment response to antidepressant medication: a prospective multi-site clinical trial. Ment Health Relig Cult. 2011;14(8):805-18. http://dx.doi.org/10.1080/1 3674676.2010.527931. PMid:22736954.

10. Lucchetti G, Lucchetti AL, Espinha DC, Oliveira LR, Leite JR, Koenig HG. Spirituality and health in the curricula of medical schools in Brazil. BMC Med Educ. 2012;12(1):78. http://dx.doi.org/10.1186/1472-6920-12-78. PMid:22900476.

11. Koenig HG, Hooten EG, Lindsay-Calkins E, Meador KG. Spirituality in medical school curricula: findings from a national survey. Int J Psychiatry Med. 2010;40(4):391-8. http://dx.doi.org/10.2190/PM.40.4.c. PMid:21391410.

12. Neely D, Minford EJ. Current status of teaching on spirituality in UK medical schools. Med Educ. 2008;42(2):176-82. http:// dx.doi.org/10.1111/j.1365-2923.2007.02980.x. PMid:18230090.

13. Puchalski CM. Integrating spirituality into patient care: an essential element of person-centered care. Pol Arch Med Wewn. 2013;123(9):491-6. http://dx.doi.org/10.20452/pamw.1893. PMid:24084250.

14. Bruno LFC. Levantamento da qualidade de vida no trabalho. [monografia]. Manaus: Universidade Federal do Amazonas; 1999.

15. Schmidt MJ. Understanding and using statistics base concepts. Massachusetts, USA: D.C. Health and company; 1975.

16. Lucchetti G, Oliveira LR, Koenig HG, Leite JR, Lucchetti AL. Medical students, spirituality and religiosity: results from the multicenter study SBRAME. BMC Med Educ. 2013;13(1):162. http://dx.doi.org/10.1186/1472-6920-13-162. PMid:24314327. 


\section{iighe}

17. Lucchetti G, Lucchetti AL. Spirituality, religion, and health: over the last 15 years of field research (1999-2013). Int J Psychiatry Med. 2014;48(3):199-215. http://dx.doi.org/10.2190/PM.48.3.e. PMid:25492714.

18. Moreira-Almeida A, Koenig HG, Lucchetti G. Clinical implications of spirituality to mental health: review of evidence and practical guidelines. Rev Bras Psiquiatr. 2014;36(2):176-82. http://dx.doi.org/10.1590/1516-4446-2013-1255. PMid:24839090.

19. Paal P, Roser T, Frick E. Developments in spiritual care education in German - speaking countries. BMC Med Educ. 2014;14(1):1-7. http://dx.doi.org/10.1186/1472-6920-14-112. PMid:24898431.

20. Borneman T, Ferrell B, Puchalski CM. Evaluation of the FICA tool for spiritual assessment. J Pain Symptom Manage. 2010;40(2):163-73. http://dx.doi.org/10.1016/j.jpainsymman.2009.12.019. PMid:20619602.

21. Lewis S, Salins N, Rao MR, Kadam A. Spiritual well-being and its influence on fatigue in patients undergoing active câncer directed treatment: a correlational study. J of Canc Res and Therap. 2014;10(3):676-80. PMid:25313759.

22. Puchalski CM. Spirituality and medicine: curricula in medical education. J Cancer Educ. 2006;21(1):14-8. http://dx.doi. org/10.1207/s15430154jce2101_6. PMid:16918282.

23. Rouleau CR, Garland SN, Carlson LE. The impact of mindfulness-based interventions on symptom burden, positive psychological outcomes, and biomarkers in cancer patients. Cancer Manag Res. 2015;7:121-31. PMid:26064068.

24. Damiano RF, Costa LA, Viana MTSA, Moreira-Almeida A, Lucchetti ALG, Lucchetti G. Brazilian scientific articles on "Spirituality, Religion and Health". Arch Clin Psychiatry. 2016;43(1):11-6. http://dx.doi.org/10.1590/0101-60830000000073.

\section{Contribuição dos autores}

Simone Regina Souza da Silva Conde participou da elaboração do projeto de pesquisa, Coleta e análise de dados, elaboração do artigo científico. José Heitor Borburema de Oliveira participou da coleta e análise de dados. Ulisses Tavares de Arruda participou da coleta e análise de dados. Luis Eduardo de Carvalho Barros participou da coleta e análise de dados. Sylvia Helena Souza da Silva Batista participou da concepção da pesquisa, da elaboração e revisão do manuscrito. Nildo Alves Batista participou da concepção e da supervisão da pesquisa, da análise e interpretação dos dados e da elaboração e revisão do manuscrito. 